\title{
Final Report \\ DOE Grant No. DE-FG03-01ER54617 \\ Computer Modeling of Microturbulence and Macrostability Properties of Magnetically Confined Plasmas
}

Jean-Noel Leboeuf, Principal Investigator (leboeuf@physics.ucla.edu)

Viktor K. Decyk, Co-Principal Investigator (deyck@physics.ucla.edu)

Department of Physics and Astronomy

University of California

Los Angeles, CA 90095-1547

Grant Period: January 15, 2001-January 14, 2004.

\section{Executive Summary}

We have made significant progress during the past grant period in several key areas of the UCLA and national Fusion Theory Program. This impressive body of work includes both fundamental and applied contributions to MHD and turbulence in DIII-D and Electric Tokamak plasmas, and also to Z-pinches, particularly with respect to the effect of flows on these phenomena. We have successfully carried out interpretive and predictive global gyrokinetic particle-in-cell calculations of DIII-D discharges. We have cemented our participation in the gyrokinetic PIC effort of the SciDAC Plasma Microturbulence Project through working membership in the Summit Gyrokinetic PIC Team. We have continued to teach advanced courses at UCLA pertaining to computational plasma physics and to foster interaction with students and junior researchers. We have in fact graduated $2 \mathrm{Ph}$. $\mathrm{D}$. students during the past grant period. The research carried out during that time has resulted in many publications in the premier plasma physics and fusion energy sciences journals and in several invited oral communications at major conferences such as Sherwood, Transport Task Force (TTF), the annual meetings of the Division of Plasma Physics of the American Physical Society, of the European Physical Society, and the 2002 IAEA Fusion Energy Conference, FEC 2002. Many of these have been authored and co-authored with experimentalists at DIII-D.

\section{Significant Accomplishments During Past Grant Period}

[Nota Bene: Herein and in the Bibliography, references to group work during the past grant period are denoted by LD, references to other work by $\mathrm{R}$ and references to group work prior to the past grant period are denoted by RLD]

The UCLA Fusion Plasma Simulation Group has become significantly smaller with the untimely death of John Dawson on November 17, 2001. The group which now consists of two senior members of the UCLA research faculty, Viktor Decyk and Jean-Noel Leboeuf, joined in June 2002 by one Ph. D. Student, James Kniep, has more than 50 years of cumulative experience in computational plasma physics. The group holds many of the inventions associated with modern particle-in-cell (PIC) simulation techniques, particularly with regards to the parallel implementation of these models. This particle code expertise has been augmented by the return to UCLA in fall 1998 of Jean-Noel Leboeuf who brought back with him considerable know-how in parallel fluid, and MHD models of plasmas. The group is now the repository of the FAR suite of MHD codes originally developed at ORNL and which have been used at UCLA for benchmarks performed as part of NIMROD Team activities and for studies of flow effects on MHD instabilities; of an array of global gyrofluid models of plasma turbulence which are useful for fast linear scoping of realistic, finite beta, shaped, magnetic equilibria and may prove well suited in the nonlinear mode for integration endeavors; and of a global toroidal gyrokinetic particle code, the Ucla-CANada (UCAN) 
code limited to circular geometry and adiabatic electrons but still useful for experimental predictions and interpretations for UCLA's own Electric Tokamak (ET) and for DIII-D at General Atomics. The group has also been actively involved in the development of a general geometry, global, toroidal, three-dimensional, gyrokinetic PIC code with mobile electrons and electromagnetic effects, the Summit Gyrokinetic Framework, as part of the SciDAC Plasma Microturbulence Project. In spite of its small size, the group has been very productive in its physics and computational physics contributions to plasma stability and transport in magnetic fusion devices, particularly during the past 3-year grant period (16 refereed journal publications, 9 conference proceedings contributions and 11 invited communications). Progress realized in the areas of MHD, turbulence, and development of computational models and techniques is detailed in what follows.

1) Effect of sheared flows on MHD stability and turbulence of magnetically confined plasmas: One major recurring theme in our research has been thorough studies of the effect of externally imposed and self-consistently generated flows on MHD stability and turbulence of magnetically confined plasmas. It has clearly been one of the main preoccupations of the US fusion program [R1, R2, R3]. Our work offers yet another proof of how beneficial sheared flow can be to increase plasma stability and reduce plasma turbulence.

With respect to ET we have shown through linear calculations with the FAR suite of MHD codes that externally imposed sheared poloidal flows will be more effective in stabilizing MHD instabilities such as resistive internal kinks (chosen as a paradigm) than sheared toroidal flows. Poloidal flows on the order of the poloidal sound speed can lead to stabilization [LD2]. On the other hand, toroidal flows, included perturbatively in the stability

calculations, have either no effect in the case of rigid rotation or lead to destabilization through an increase in the effective plasma beta in the case of sheared rotation [LD6]. Similarly, global gyrokinetic calculations using UCAN with model ET parameters and profiles have shown that poloidal sheared flows on the order of the poloidal sound speed can suppress ion temperature gradient (ITG) turbulence (also chosen as a paradigm) [RLD4] while toroidal sheared flows on the order of the Alfven speed only lead to a significant reduction [LD6]. We regard these observations as major contributions to the ET program even though only a "regime change" may see them being put to a test in the ET experiment. Another significant contribution to the ET program has been the pursuit of ultra-high beta equilibria with high plasma diamagnetism [R5]. Equilibrium calculations performed in the flux-conserving mode with the TOQ (GA) and RSTEQ (ORNL) Grad-Shafranov equilibrium solvers have so far fallen short of that lofty mark but do indicate a favorable poloidal beta limit which scales like 1+A, where $A$ is the aspect ratio [LD13]. This result has recently been confirmed in the case of low aspect ratio, highly shaped plasmas through equilibrium calculations using TOQ performed at GA [R6]. Both sets of results still augur well for high plasma beta operation.

Getting back to flows, with respect to DIII-D, we have shown through global gyrokinetic UCAN calculations of ITG driven turbulence with and without self-generated flows that the zonal flows are essential in regulating the radial correlation length of the turbulence [RLD7, LD17], in agreement with the more generic result of Lin et al. [R8]. For parameters and profiles appropriate to DIII-D LMode discharges, the radial correlation length in the calculations is on the order of the poloidal ion Larmor radius or at least several times the ion Larmor radius itself, with zonal flows. It is much longer, more on the order of a fraction of the minor radius, without zonal flows. The correlation length diagnostic in the calculations has been made as close to the experimental one as possible to make the comparisons even more meaningful. It is a first attempt at a synthetic diagnostic. We emphasize that our gyrokinetic calculations with circularized DIII-D profiles and parameters have reproduced the trend as well as the size of the radial correlation length as a function of minor radius measured experimentally [RLD9].

MHD activity can generate flows too. In fact, our MHD calculations performed with the FAR suite of codes and pertaining to resistive interchange modes in DIII-D discharges with negative central 
shear (NCS) have again shown that poloidal flows generated nonlinearly by the fluctuations themselves through Reynolds stress have a very different character in cylindrical and toroidal geometry [LD5]. Remarkably, these zonal flows with toroidal mode number $n=0$ can have a strong poloidal localization in toroidal geometry as opposed to cylindrical geometry where the flows are poloidally uniform. Put another way, toroidally uniform $n=0$ zonal flows with poloidal mode number $m=1$ appear to be preferentially excited in toroidal geometry whereas only $m=0$ flows are possible in cylindrical geometry. This confirms anew the importance of toroidicity for the structure of zonal flows which we first encountered in more generic reduced MHD calculations [RLD10]. This is a significant contribution to the physics ingredients of a characterization and control strategy of MHD instabilities, particularly where few other knobs are available such as in ITER-class burning plasmas. We also note that this activity has led to a collaboration with JAERI, Naka, Japan, which consists in modeling MHD activity in JT60-U discharges with deeply reversed shear using the FAR suite of codes. In contrast to DIII-D, these JT60-U discharges appear to be more susceptible to double tearing modes than interchange modes, with flows again playing a crucial role in their nonlinear saturation [LD15, LD24].

We have also studied the effect of externally imposed sheared flow, in this case axial sheared flow, on the MHD stability of Z-pinches in preparation for experiments at the Nevada Terawatt Facility. In collaboration with colleagues from the University of Nevada at Reno we have developed a comprehensive linear eigenvalue solver for a set of ideal MHD equations appropriate for Z-pinches with axial sheared flow, axial magnetic field, and with the Hall term. We have shown that axial sheared flow is an effective stabilizer of sausage and kink modes particularly in the presence of the Hall term which by itself tends to exacerbate these instabilities [LD8]. These linear extended MHD results have since been confirmed by electromagnetic, nonlinear, three-dimensional hybrid calculations with particle ions and massless fluid electrons starting from a Z-pinch MHD equilibrium [LD16, LD20, LD22]. An example of a Z-pinch discharge stabilized (quieted) by axial sheared flow from these hybrid calculations is shown in the accompanying figure.
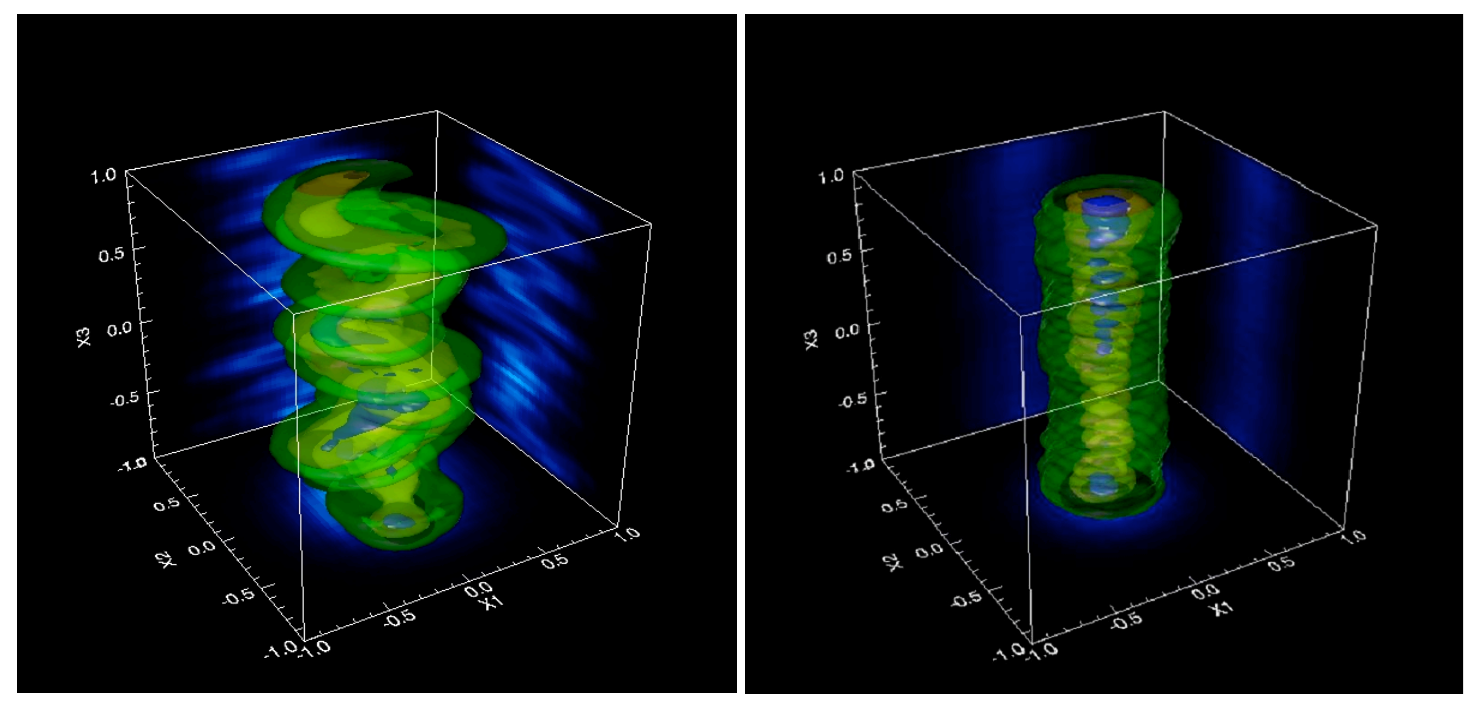

Figure Caption: Potential contours in the late nonlinear stage of the hybrid calculations are shown on the left without axial sheared flow (violent) and on the right with (quiet).

2) Interpretive and predictive gyrokinetic particle-in-cell calculations of turbulence in DIII-D-like discharges: 
We were one of the first groups (see also R11) to aggressively (some even said prematurely) apply global gyrokinetic simulations to actual experiments in spite of their limitations to conventional ITG. So doing, we have been blessed with a strong and productive interaction with experimentalists at DIII-D, particularly Curt Rettig (since departed) and Terry Rhodes. The primary physics goal has been characterization, understanding and control of ion channel turbulence. This rather intense and longstanding collaboration has resulted in a body of work which is both predictive and interpretive of plasma behavior in DIII-D discharges.

With respect to interpretation, in addition to the successful comparisons of the radial correlation lengths measured experimentally and calculated for L-mode discharges in DIII-D described earlier, we have used our global gyrokinetic PIC code UCAN to model several other types of experimental DIII-D discharges. These include those specifically tailored to exacerbate ITG turbulence and for which we were able to show that the calculations exhibit ITG activity under the discharge conditions in which it was experimentally inferred. This was reported in an Invited Talk by Curt Rettig at the Quebec DPP/APS 2000 meeting and in a subsequent publication co-authored with experimentalists [LD4]. Calculations were also performed with profiles and parameters appropriate for Quiescent Double Barrier or QDB discharges. Remarkably enough, the lack of trend in the radial correlation lengths as a function of minor radius appears to be reproduced by the calculations [LD29, LD30]. In discharges where comparisons could be made, computations with UCAN and experiments have also shown good agreement for radial and poloidal wavenumber spectra and for the magnitude and radial trend of the density fluctuations. This body of work was presented in an invited talk at the 2001 APS/DPP meeting by Terry Rhodes [LD33] and in several publications authored or coauthored by DIII-D experimentalists [LD7, LD9, LD10, LD17, LD23].

Recently, the favorable comparisons between UCAN computations and experimental correlation length measurements have been corroborated by gyrofluid Gryffin, gyrokinetic Vlasov or continuum GS2 and GYRO computations for DIII-D self-similar discharges with the computed and measured radial correlation lengths agreeing to better than a factor 2 [LD23].

In addition to these interpretive calculations, we have successfully used our global gyrokinetic UCAN calculations to make predictions about the outcome of an experiment on DIII-D which we jointly proposed with experimentalists. Previous experiments and gyrokinetic calculations [RLD7, RLD9] appeared to show that radial correlation lengths of the turbulence in typical DIII-D L-mode discharges scale with the poloidal Larmor radius or at least are several times the Larmor radius instead of following the Larmor radius itself. We realized that this could be explored further by changing the poloidal magnetic field. This could be accomplished experimentally by adjusting the current so as to lower or raise q, and computationally by just shifting the whole q profile up and down or by changing the aspect ratio. Computations performed prior to the experiments and in which the q-profile was changed by a factor of 4 showed little evidence of the expected commensurate and large change in the radial correlation length [LD29, LD30]. This is illustrated in the accompanying figure. The curves represent the well separated poloidal Larmor radius with lowered q (red; small poloidal Larmor radius), base q (blue; intermediate poloidal Larmor radius) and raised q (green; large poloidal Larmor radius. The figure does however show that there is a large degree of overlap between the calculated radial correlation lengths represented by red (lowered q) and green data points (raised q). 


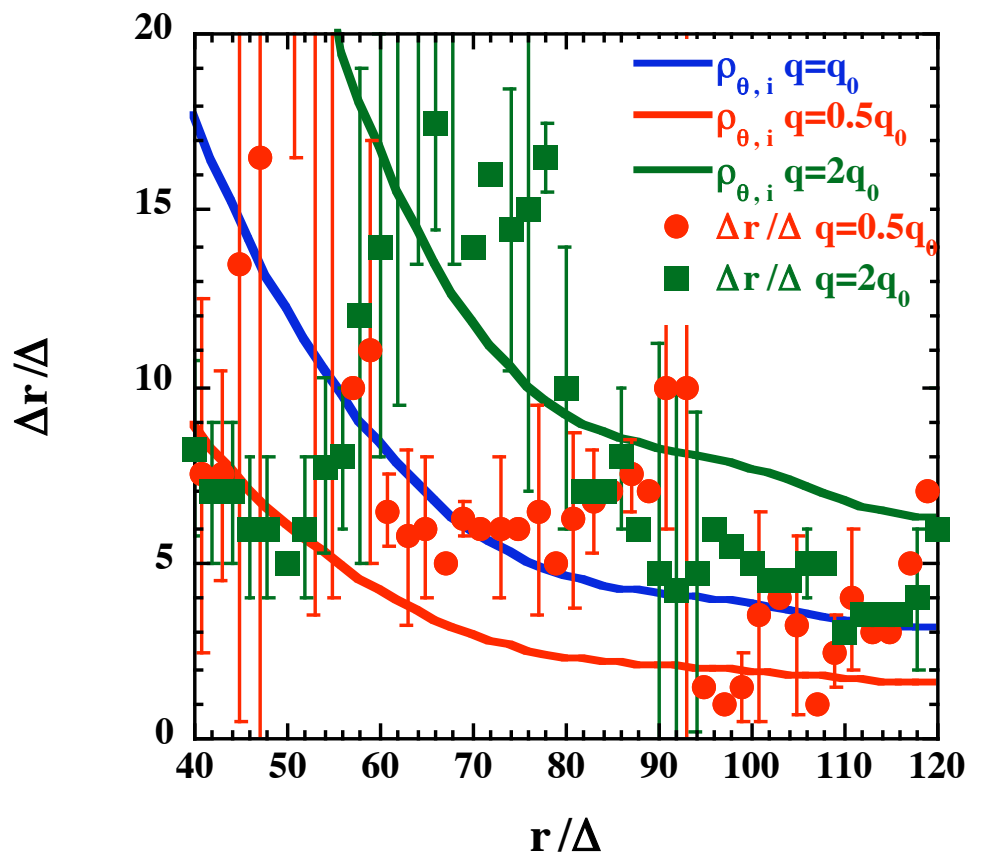

Figure Caption: Lack of definite change (green and red data points essentially overlap) in the calculated radial correlation length plotted as a function of minor radius upon significant changes in the poloidal Larmor radius (red, blue and green curves)

Apparently, it is also what has since been observed experimentally with the caveat that the q profile could only by changed by $40 \%$ in this series of experiments. The modeled aspect ratio scan, which is difficult to realize experimentally in the same device, did show a more promising trend whereby the radial correlation lengths would definitely be larger in a large aspect ratio device like ET compared to DIII-D and this by a factor corresponding roughly to the ratio of the aspect ratios [LD29]! This remains to be verified by comparative experimental measurements. A good part of this work was also featured in the invited talk delivered by Terry Rhodes at the 2001 DPP/APS meeting [LD33] and in the accompanying publication [LD9] and in his 2002 IAEA presentation [LD23].

\section{3) Computational Advances:}

The exciting physics results just described would not have been possible without the solid yet ever evolving computational foundation that has also been, is and will be, the trademark of the UCLA Fusion Plasma Simulation Group.

\section{a) General PIC Framework}

Although there are many definitions of a framework, the working definition we have adopted is that a framework is a unified environment containing all the components needed for writing code for a specific problem domain. The idea of a general PIC Framework originated from the fact that there are many different types of particle-in-cell codes in development and in use at any given time in the Plasma Physics Group at UCLA. A general PIC Framework would then cut down overall duplication between different applications (e.g., laser, beam, accelerator, process, space, fusion 
plasmas), insure maximum re-use of common modules, facilitate code modernization and diagnostic development, and accelerate enhancement of code performance [LD31].

This Framework has been designed to provide high quality object-based components for parallel PIC codes and allow rapid development of new PIC codes. This Framework is currently being used in QuickPIC, a quasi-static laser-plasma acceleration code [LD19], and BEPS, an electrostatic code used for teaching plasma physics. It is also a prototype for the Summit Gyrokinetic Framework [LD14, LD25]. Recent developments have added electromagnetic field solvers to the Framework, which would support both fully electromagnetic codes as well as Darwin codes, along with a variety of boundary conditions for fields and particles.

The portable library of particle and field management routines PLIB developed at UCLA and in wide use throughout the fusion, space and accelerator plasmas communities has been upgraded to two-dimensional domain decomposition from the one-dimensional one it was limited to previously. This also applies to the Fast Fourier Transform (FFT) routines within PLIB. The one-dimensional decomposition, while natural for certain problems, sets limits on how large a problem can be run because of the available memory per node. The more flexible P2LIB permits larger problems to be run on a larger number of processors.

With P2LIB, the Framework codes have in fact run efficiently with up to 2048 nodes on the NERSC SP3/375. An electrostatic code with one billion particles and 2048 nodes achieves about $65 \%$ parallel efficiency, running about 1 microsecond/particle/processor. This is particularly attractive for NERSC's Phase III IBM-SP which will have in excess of 4000 processors. The accompanying figure illustrates that the performance obtained on NERSC's IBM-SP with 2D domain decomposition for a 3D full dynamics PIC benchmark is close to ideal speedup and this augurs well for performance improvements on the Phase III SP. 


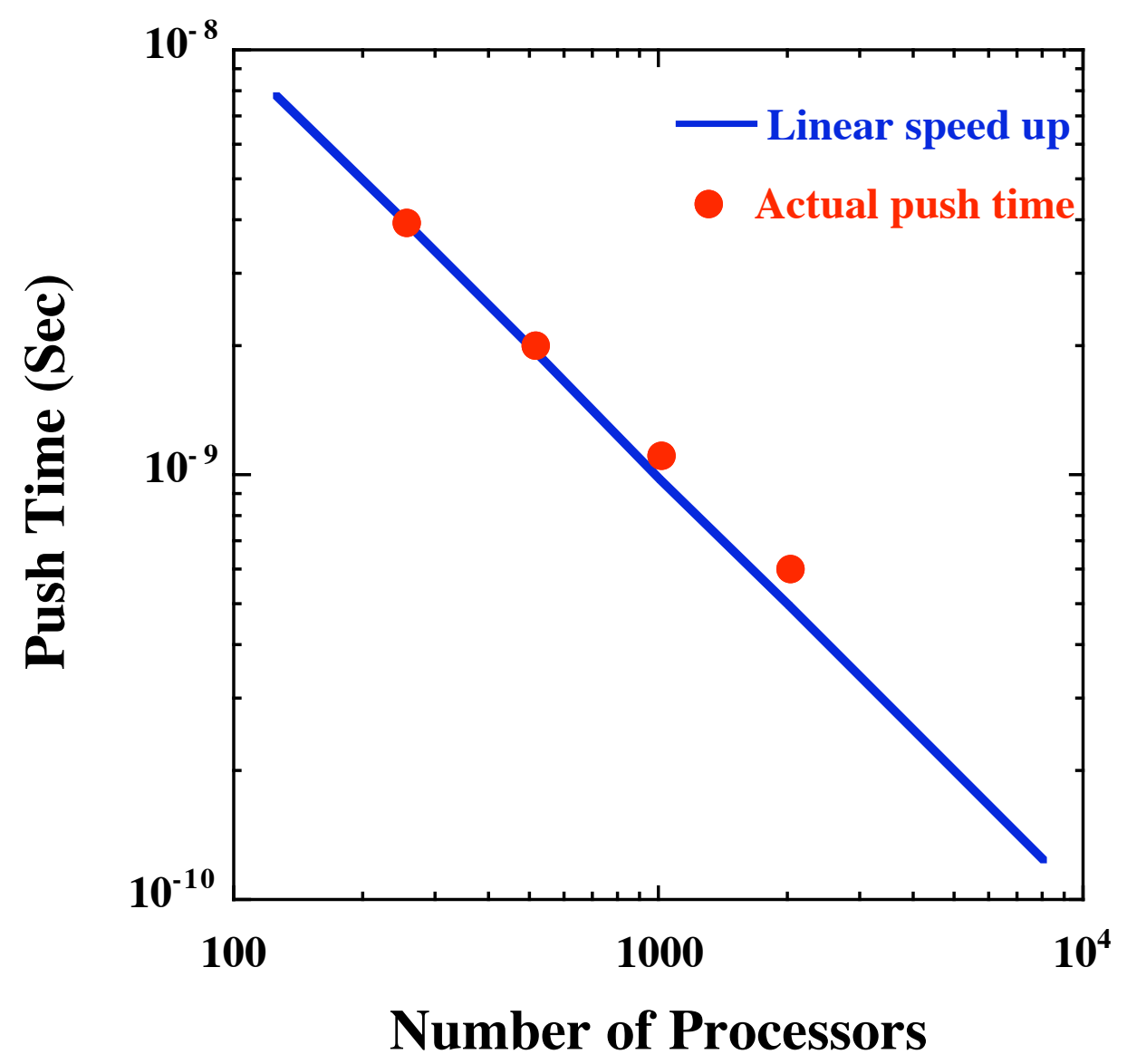

Figure Caption: Near linear speedup obtained with general PIC framework code up to 2048 processors on the IBM-SP at NERSC.

Although this is an outstanding result, we are continuing to study ways to improve the performance of such very large systems.

b) Joint gyrokinetic PIC code modernization and joint gyrokinetic PIC code development within the Summit Gyrokinetic Framework

Based on its experience with the general PIC Framework, UCLA has provided continuing guidance in the conception of the Summit Gyrokinetic Framework [http://www.nersc.gov/scidac/summit/] and actively participated in its implementation. The Summit Gyrokinetic PIC Framework is part of the four-pronged computational arsenal in the SciDAC Plasma Microturbulence Project along with the PPPL/UC-Irvine global gyrokinetic code GTC [R8] on the PIC or discrete side of the project and the Vlasov flux tube GS2 (U. Maryland/Texas) code [http://gs2.sourceforge.net/] and global GYRO (GA) code [http://web.gat.com/comp/parallel/gyro.html] on the continuum side of the same project.

The building blocks of the Summit Gyrokinetic PIC Framework have been existing modules such as the LLNL (pg3eq), UCLA (UCAN) and U. Colorado (TUBE) codes which had to be 
modernized before integration. While UCAN is global in Cartesian coordinates, pg3eq uses the quasi-ballooning representation of a thin toroidal annulus [R12] and TUBE employs a more local flux tube implementation. Electrons and electromagnetic effects have recently been successfully included in Summit through TUBE [LD14, LD25].

The modernization effort has consisted in using features of FORTRAN90 to encapsulate these eminently reusable and very useful legacy FORTRAN77 codes through wrappers and interfaces [LD27]. It has resulted in much more robust, usable, more easily modified, and portable modules. These pieces of the overall Summit Gyrokinetic Framework are being further integrated through the use of a common main, common input deck, and common definitions of classes and objects [LD14, LD25, LD31]. As of now, the modernized flux tube TUBE and quasi-ballooning pg3eq modules are accessible and executable from a common main.

A major part of the UCLA task has consisted in reviving, debugging and incorporating the noncircular version of the pg3eq module, known as pg3eq_nc, in the Summit Gyrokinetic Framework. The pg3eq_nc module was itself modernized using the same prescriptions as the other modules and message passing was implemented using MPI for its use on current massively parallel platforms [LD34, LD36].

This collaborative activity, which is leveraged at the $10 \%$ level by the SciDAC Plasma Microturbulence Project, was consolidated during several working meetings at the Sherwood, APS and ISOFS conferences and at the participating institutions over the past grant period.

4) Other accomplishments:

One of the advantages of a university environment is that it fosters application of one's capabilities in areas which do not appear linked to the main thrust of one's program but which nevertheless contribute to its growth and consolidation through student involvement, unexpected collaborations and novel applications. The efforts described below are examples of such leverage.

a) Global gyrofluid calculations of ITG turbulence: All along, our trademark has been global calculations of turbulence and this work pertains to global 3D gyrofluid calculations of ITG turbulence [RLD13], here in cylindrical geometry but which include ion Landau damping. Apart from maintaining a desirable computational capability which has unfortunately all but disappeared elsewhere in the US program, the physics goal of this global gyrofluid study is characterization of ITG turbulence as the ratio of sound Larmor radius to plasma minor radius, also called normalized sound Larmor radius, denoted by $\square_{*}$ is changed. These recently published calculations [LD1] show

that the saturated levels of turbulence, the steady-state poloidal wavenumber spectra and the heat diffusivities scale with $\square_{*}$ and therefore obey a gyro-Bohm scaling even though the temperature and density profiles are close to marginal. This both agrees and disagrees with other calculations performed in toroidal geometry. As it is, this scaling with $\square_{*}$ is very favorable for a large device such as ITER, as are recent global gyrokinetic calculations of Lin et al. [R14]. The accompanying figure clearly demonstrates the increasing scale sizes of the temperature fluctuations in the poloidal plane with increasing $\square_{*}$. 


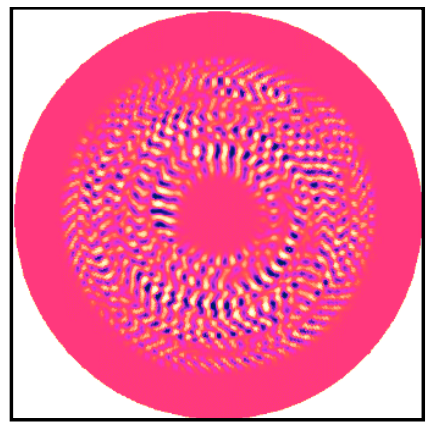

$\square_{*}=0.008$

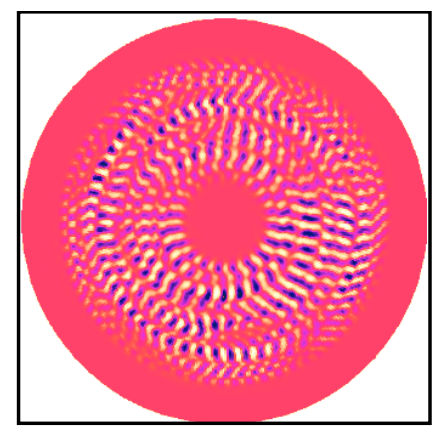

$\square * 0.010$

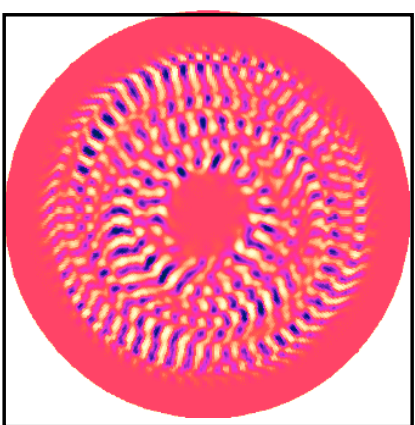

$\square_{*}=0.012$

Figure Caption: Contours of the temperature fluctuations in the poloidal plane with increasing $\square_{*}$.

b) LDX: We have taken the excuse of a computational physics class and of a $\mathrm{Ph}$. $\mathrm{D}$. thesis to study the kinetic stability of the very interesting magnetic dipole configuration using classic PIC simulations. This configuration is the object of the Levitated Dipole Experiment (LDX). The simulation effort has concentrated on the simpler problem of the stability to interchange modes of a plasma confined by the magnetic field of a long straight wire or straightened levitated ring. We have used the fully electromagnetic PIC code $\mathrm{P}^{3}$ arsec, developed by John Tonge for his $\mathrm{Ph}$. D. thesis to simulate such plasmas. Our conclusion is that kinetic stability closely tracks that given by the

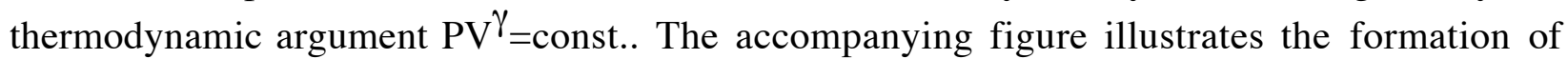
convective cells in the electrostatic potential due to interchange instability for profiles which should be unstable (according to the thermodynamic and kinetic criteria) to such modes with $\mathrm{n}(\mathrm{r}) \sim \mathrm{r}^{-2}$ and $\mathrm{T}(\mathrm{r}) \sim \mathrm{r}^{-2}$ and $\mathrm{B}(\mathrm{r}) \sim 1 / \mathrm{r}$ (for a long straight wire).
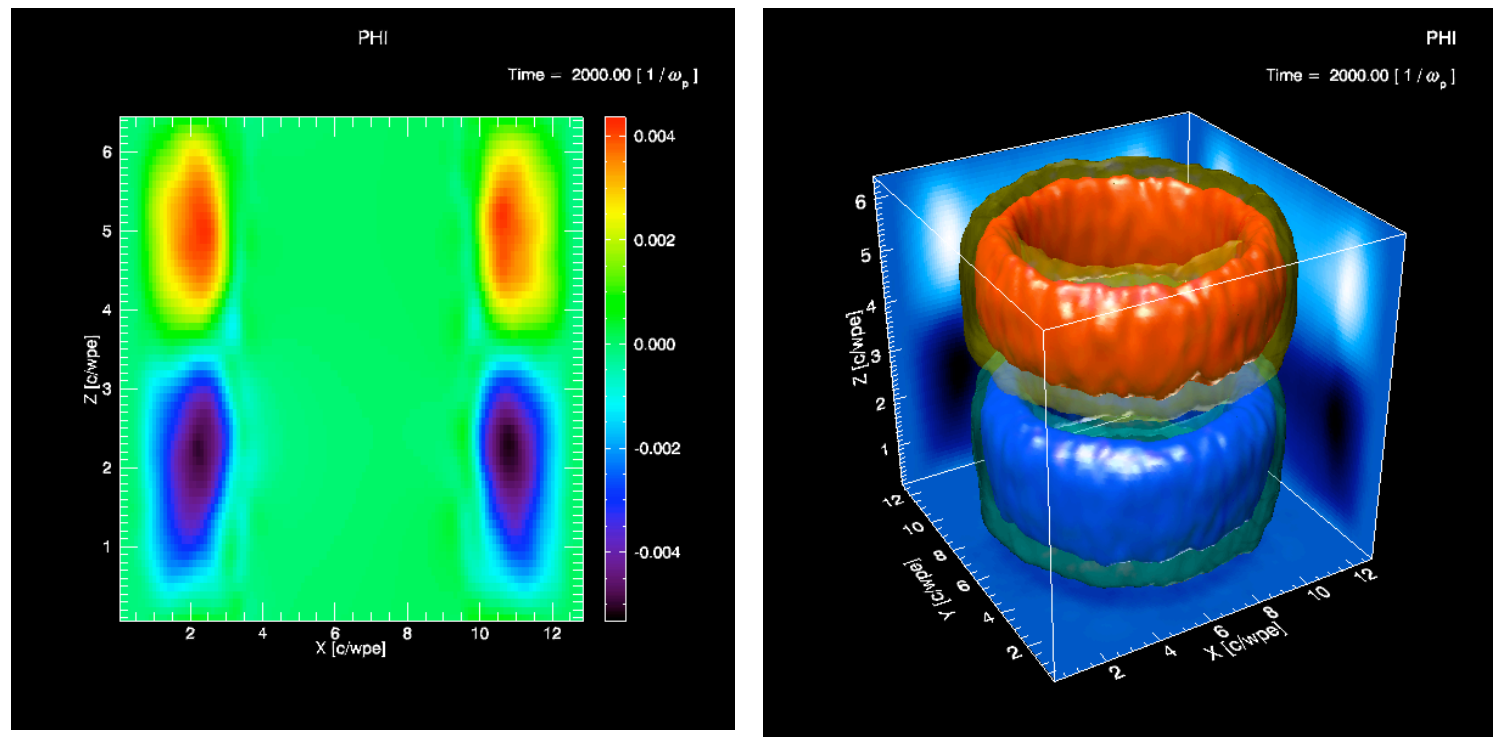

Figure Caption: Convective cells due to interchange instability of plasma confined in the magnetic field of a long straight wire.

c) Assessment of collisional models for kinetic susceptibilities: Inclusion of collisions in computational models is always problematic because there are few analytical expressions to compare with and few numerical studies of the effects of collisions on the kinetic normal modes of the plasma. We have recently completed an assessment of Krook collisional models. We found that not only the energy and number conserving operators but also the number conserving Krook 
operator are adequate to capture collisional effects in the kinetic regime compared to the Lorentz and Lenard-Bernstein collision operators which are more complicated to implement and calculate[LD11]. This effort will help greatly in guiding inclusion of collisions in our particle models.

d) Dynamics of a supersonic plume moving along a magnetized plasma: The propagation of a plasma plume, upon pellet ablation for instance, through a magnetized plasma is not only of interest for fusion applications but also from a generic plasma physics standpoint. We have begun particlein-cell simulation studies [LD12, LD21] of this phenomenon. For scale lengths representative of both laboratory and auroral phenomena, the major nonlinear effects identified by the present simulations are the formation of a bipolar current system from the ballistic electrons, the appearance of transient potential layers, and the carving of deep density cavities. Also, a 3D magnetic topology is generated by the self-consistent ballistic and diamagnetic currents that accompany highly localized potential layers. If anything, this work has relevance to fueling of ITER-class plasmas which are bound to be as collisionless as the ones simulated here.

\section{5) Synergistic activities}

Many of the achievements during the past grant period would not have been possible without the synergistic activities to be described next, specially without the development and deployment of our local AppleSeed parallel cluster of Macintosh computers.

\section{a) Cluster computing}

Viktor Decyk, with help from UCLA Ph. D. student Dean Dauger who degreed in June 2001, has continued to develop, upgrade, update and disseminate the web-downloadable [http://exodus.physics.ucla.edu/appleseed/dev/developer.html] software needed to set-up a parallel cluster using Macintosh computers such as UCLA's own G4 AppleSeed cluster [LD18, LD32, LD35]. We are actively using the AppleSeed cluster for teaching and research. It has proven invaluable in a classroom environment because of its ease of use by the students and because of the turnaround it can provide on a class time scale.

b) Education and outreach highlights

Viktor Decyk became the first Distinguished Lecturer under the auspices of the newly constituted Plasma Science and Technology Institute (PSTI) at UCLA. As such he gave a series of weekly lectures spanning the full duration of the Spring 2002 quarter on the topic of "Scientific computing with FORTRAN95". More details on the lectures can be found at:

http://exodus.physics.ucla.edu/Fortran95/PSTIResearchLecSeries1.html

James Kniep, a graduate student in the UCLA Department of Physics and Astronomy, has started working in the Fusion Plasma Simulation Group as part of his $\mathrm{Ph}$. D. program. The current topic of his research concerns modifications due to strong poloidal flows and parallel nonlinearities of the gyrokinetic ion orbits and their implications for gyrokinetic particle simulations of ITG driven turbulence. James Kniep actively uses our local parallel AppleSeed cluster of Macintosh G4 computers.

The Fusion Plasma Simulation Group and UCLA's Plasma Science and Technology Institute (PSTI) hosted Professor Hiroshi Naitou from Yamaguchi University in Ube, Japan, from August 1, 2002 to September 30, 2002, under the auspices of the USDOE-Japan Ministry of Education, Culture, Sports, Science and Technology (MEXT) Joint Institute for Fusion Theory (JIFT) exchange program in fusion theory and computations. 
During the past 2 years, two students supervised by members of the group obtained their Ph. D.. Both have remained at UCLA. Pierre-Alexandre Gourdain, from the Electric Tokamak, received the Doctorat es Sciences, in December 2001, CEGELY, from Ecole Centrale de Lyon, 36 Avenue Guy de Collongue, 69131 Ecully Cedex, France. His Doctoral Thesis was entitled: "Resolution de l'equilibre axisymmetrique de MHD ideale avec frontiere libre en utilisant un principe variationel" and tackled the timely topic of numerical computations of high beta equilibria discussed earlier with applications to ET. John Tonge received a Ph. D., in Physics, in May 2002, from UCLA. His Doctoral Thesis was entitled: "Particle Simulations of Instabilities in Space and Astrophysical Plasmas", with a chapter devoted to interchange instabilities related to LDX, results from which were briefly described earlier.

c) Community service highlights

Jean-Noel Leboeuf was a member of the Program Committee, representing Magnetic Confinement Theory, 43rd Annual meeting of the Division of Plasma Physics, American Physical Society, Long Beach, CA, October 29 - November 2, 2001. He is currently serving on the FESAC subpanel "Integrated Simulation and Optimization of Fusion Systems" or ISOFS. More information on this activity can be found at: http://www.isofs.info/. He has recently been appointed by the US and Japan to the JIFT Steering Committee.

Viktor Decyk and Jean-Noel Leboeuf were the local organizers and members of the program committees for the US-Japan Workshop on Simulations of Plasmas held at UCLA on May 16 and 17, 2002 and for the Dawson Symposium held at UCLA right after the Workshop on May 18, 2002.

d) websites

Reports on the fusion plasma simulation activities at UCLA can be found at: http://exodus.physics.ucla.edu/UCLAPlasmaSimulation.html.

UCLA activities relevant to PSACI's and SciDAC's Plasma Microturbulence Project (PMP) can be found at:

http://fusion.gat.com/theory/pmp/

UCLA activities relevant to the Gyrokinetic Summit Team of SciDAC's PMP can be found at:

http://www.nersc.gov/scidac/summit/ 


\section{BIBLIOGRAPHY}

\section{Literature Cited (Including Group Publications Before Past Grant Period)}

R1. K. H. Burrell, "Effects of EXB velocity shear and magnetic shear on turbulence and transport in magnetic confinement devices", Phys. Plasmas 4, 1499-518 (1997).

R2. P. W. Terry, "Suppression of turbulence and transport by sheared flow", Rev. Mod. Phys. 72, 109-65 (2000).

R3. P. H. Diamond, M. N. Rosenbluth, E. Sanchez, C. Hidalgo, B. Van Milligen, T. Estrada, B. Branas, M. Hirsch, H. J. Hartfuss, B. A. Carreras, "In search of the elusive zonal flow using crossbicoherence analysis", Phys. Rev. Letts. 84, 4842-4845 (2000).

RLD4. M. W. Kissick, J.-N. Leboeuf, S. C. Cowley, J. M. Dawson, V. K. Decyk, P.-A.

Gourdain, J.-L. Gauvreau, P. A. Pribyl, L. W. Schmitz, R. D. Sydora, and G. R. Tynan, "Radial Electric Field Required to Suppress Ion Temperature Gradient Modes in the Electric Tokamak" , Phys. Plasmas 6, 4722-4727 (1999).

R5. S.C. Cowley, P.K. Kaw, R.S. Kelley, and R.M. Kulsrud, "An analytic solution of high-beta equilibrium in a large aspect ratio tokamak", Phys. Fluids B 3, 2066-2077 (1991).

R6. Y. R. Lin-Liu, A. D. Turnbull, R. L. Miller, V. S. Chan, and P. A. Politzer, "A class of high $\square p$ equilibria in strongly shaped finite aspect ratio tokamak plasmas", Phys. Plasmas 10, 790-796 (2003).

RLD7. J.-N. Leboeuf, J. M. Dawson, V. K. Decyk, M. W. Kissick, T. L. Rhodes, and R. D. Sydora, "Effect of externally imposed and self-generated flows on turbulence and magnetohydrodynamic activity in tokamak plasmas", Phys. Plasmas 7, 1795-1801 (2000).

R8. Z. Lin , T. S. Hahm, W. W. Lee, W. M. Tang, and R. B. White, "Turbulent transport reduction by zonal flows: massively parallel simulations", Science 281, 1835-1837 (1998).

RLD9. T. L. Rhodes, J.-N. Leboeuf, R. Sydora, E. J. Doyle, R. A. Moyer, C. L. Rettig, K. Burrell, D. M. Thomas, G. McKee, W. A. Peebles, "Turbulent radial correlation lengths in the DIII-D tokamak", Poster P2.027, 27th European Physical Society Conference on Controlled Fusion and Plasma Physics, 12-16 June, 2000, Budapest (Hungary), Europhysics Conference Abstratcs (ECA ), Vol. 24B (2000) 564-567, European Physical Society, Petit Lancy, Switzerland. (http://sgi30.rmki.kfki.hu/EPS2000/eps2000cd/pdf/p2_027.pdf)

RLD10. E. D. Held, J. N. Leboeuf, and B. A. Carreras, "Magnetohydrodynamic calculations with a nonmonotonic q profile and equilibrium, sheared toroidal flow", Phys. Plasmas 6, 837-845 (1999).

R11. D. W. Ross, R. V. Bravenec, W. Dorland, M. A. Beer, G. W. Hammett, G. R. McKee, R. J. Fonck, M. Murakami, K. H. Burrell, G. L. Jackson, and G. M. Staebler, "Comparing simulation of plasma turbulence with experiment.", Phys. Plasmas 9, 177-184 (2002).

R12. A. M. Dimits, T. J. Williams, J. A. Byers, and B. I. Cohen, "Scalings of ion-temperaturegradient-driven anomalous transport in tokamaks", Phys. Rev. Letts. 77, 71-74 (1996). 
RLD13. L. Garcia, J. D. Alvarez, J. N. Leboeuf, V. E. Lynch, and B. A. Carreras, "Nonlinear full torus calculations of resistive-pressure-gradient-driven turbulence and ion-temperature-gradientdriven turbulence in toroidal geometry," in Fusion Energy 1998 (International Atomic Energy $\begin{array}{llllll}\text { Agency, } & \text { Vienna, } & 1999), & \text { Vol. } & 4, & \text { p . }\end{array}$ (http://www.iaea.org/programmes/ripc/physics/fec1998/html/node51.htm\#8910).

R14. Z. Lin , S. Ethier, T. S. Hahm, and W. M. Tang, "Size scaling of turbulent transport in magnetically confined plasmas", Phys. Rev. Letts. 88, 195004/1-4 (2002).

R.15. T. S. Hahm, "Nonlinear gyrokinetic equations for turbulence in core transport barriers", Phys. Plasmas 3,.4658-4664 (1996).

R16. L. Villard, S. Allfrey, A. Bottino, M. Brunetti, G. L. Falchetto, V. Grandgirard, R. Hatsky, J. Nuhrenberg, A. G. Peeters, O. Sauter, S. Sorge, and J. Vaclavik, "Full Radius Linear and Nonlinear Gyrokinetic Simulations for Tokamaks and Stellarators: Zonal Flows, Applied ExB Flows, Trapped Electrons and Finite Beta", Paper IAEA-CN-94/TH/1-3 ,19th IAEA Fusion Energy Conference, 14 to 19 October 2002 , Lyon, France, IAEA, Vienna, 2002. Published electronically as ftp://ftp.iaea.org/pub/mtcd/meetings/cn94/laurent.villard_epfl.ch01.pdf.

R17. H. Naitou, T. Kobayashi, T. Kuramoto, S. Tokuda, and T. Matsumoto , "Stabilization of kinetic internal kink mode by electron diamagnetic effect", Proceedings of 9th International Toki Conference on Plasma Physics and Controlled Nuclear Fusion (ITC-9), New Frontiers in Plasma Physics, Japan Soc. Plasma Sci. \& Nucl. Fusion Res. 1999, pp.259-62. Nagoya, Japan.

R18. M. Ottaviani, and F. Porcelli, "Fast nonlinear magnetic reconnection", Phys. Plasmas 2, 41044117 (1995).

R 19. Glenn Bateman, Arnold H. Kritz, Jon E. Kinsey, Aaron J. Redd, and Jan Weiland, "Predicting temperature and density profiles in tokamaks", Phys. Plasmas 5, 1793-1799 (1998).

R 20. M.Gilmore, S. Kubota, W.A. Peebles, X.V. Nguyen, D. Stutman, and the NSTX Team, "Changes in Edge Turbulence with rho-star and Toroidal Rotation Input in NSTX", Talk presented at the 44th Annual Meeting of the APS Division of Plasma Physics, Nov 11-15, 2002, Orlando, FL, Bull Amer Phys Society 47, 60.

\section{Group Publications Cited (Past Grant Period)}

Refereed publications

LD1. J. N. Leboeuf, V. E. Lynch, B. A. Carreras, J. D. Alvarez, and L. Garcia, "Full torus Landau fluid calculations of ion temperature gradient driven turbulence in cylindrical geometry", Phys. Plasmas 7, 5013-5022 (2000).

LD2. M. W. Kissick, J. N. Leboeuf, S. C. Cowley, and J. M. Dawson, "Poloidal rotation effects on a simulated resistive kink mode", Phys. Plasmas 8, 174-179 (2001).

LD3. P. P. Sosenko, P. Bertrand and V. K. Decyk, "Krylov-Boholyubov methods and gyrokinetics", Physica Scripta 64, 264-272 (2001).

LD4. C. L. Rettig, G. M. Staebler, T. L. Rhodes, J. N. Leboeuf, W. A. Peebles, E. J. Doyle, K. H. Burrell, and R. A. Moyer, "Search for the ion temperature gradient mode in a tokamak plasma and comparison with theoretical predictions", Phys. Plasmas 8, 2232-2237 (2001). 
LD5. J. N. Leboeuf, V. E. Lynch, and B. A. Carreras, "Linear and nonlinear magnetohydrodynamic stability of tokamak discharges with negative central shear", Phys. . Plasmas 8, 3358-3366 (2001).

LD6. C. G. Hobbs, M. G. House, J. N. Leboeuf, J. M. Dawson, V. K. Decyk, M. W. Kissick, , and R. D. Sydora, "Effect of sheared toroidal rotation on ion temperature gradient turbulence and resistive kink stability in a large aspect ratio tokamak", Phys. Plasmas 8, 4849-4855 (2001).

LD7. Doyle, E.J.; Baylor, L.R.; Burrell, K.H.; Casper, T.A.; DeBoo, J.C.; Ernst, D.R.; Garofalo, A.M.; Gohil, P.; Greenfield, C.M.; Groebner, R.J.; Hyatt, A.W.; Jackson, G.L.; Jernigan, T.C.; Kinsey, J.E.; Lao, L.L.; Lasnier, C.J.; Leboeuf, J.-N.; Makowski, M.; McKee, G.R.; Moyer, R.A.; Murakami, M.; Osborne, T.H.; Peebles, W.A.; Porkolab, M.; Porter, G.D.; Rhodes, T.L.; Rost, J.C.; Rudakov, D.; Staebler, G.M.; Stallard, B.W.; Strait, E.J.; Sydora, R.D.; Synakowski, E.J.; Wade, M.R.; Wang, G.; Watkins, J.G.; West, M.P.; Zeng, L., "The quiescent double barrier regime in the DIII-D tokamak",. Plasma Physics and Controlled Fusion, vol.43, (no.12A), IOP Publishing, Dec. 2001. p.A95-A112.

LD8. V. I. Sotnikov, I. Paraschiv, V. Makhin, B. S. Bauer, J. N. Leboeuf, and J. M. Dawson, "Linear analysis of the shear flow stabilization of the global MHD instabilities based on the Hall fluid model", Phys. Plasmas 9, 913-922 (2002).

LD9. T. L. Rhodes, J.-N. Leboeuf, R.D. Sydora, R. J. Groebner, E. J. Doyle, G. R. McKee,W. A. Peebles, C. L. Rettig, L. Zeng, and G. Wang, "Comparison of turbulence measurements from DIIID L-mode and high performance plasmas to turbulence simulations and models", Phys. Plasmas 9, 2141-2148 (2002).

LD10. C. M. Greenfield, K. H. Burrell, E. J. Doyle, R. J. Groebner, W. P. West, T. A. Casper, J. C. DeBoo, C. Fenzi, P. Gohil, J. E. Kinsey, L. L. Lao, J. N. Leboeuf, M. A. Makowski, G. R. McKee, R. A. Moyer, M. Murakami, R. I. Pinsker, G. D. Porter, C. L. Rettig, T. L. Rhodes, G. M. Staebler, B. W. Stallard, E. J. Synakowski, L. Zeng and the DIII-D Team, "The quiescent double barrier regime in DIII-D", Plasma Phys. Control. Fusion 44, Supplement 5A, A123-A135 (2002).

LD11. M. Opher, G. J. Morales, and J. N. Leboeuf, "Krook collisional models of the kinetic susceptibility of plasmas", Phys. Rev. E 66, 016407 (2002).

LD12. F. S. Tsung, G. J. Morales, and J. N. Leboeuf, "Dynamics of a supersonic plume moving along a magnetized plasma", Phy. Rev. Letts. 90, 55004.(2003).

LD13. M.W. Kissick, J.-N. Leboeuf, and S.E. Kruger, "On the poloidal beta evolution of fixed-q heating in tokamaks", Phys. Plasmas 10, 1060-1065 (2003).

LD14. Y. Chen, S. E. parker, B. I. Cohen, A. M. Dimits, W. M. Nevins, D. Shumaker, V. K. Decyk, and J. N. leboeuf, "Simulations of turbulence transport with kinetic electrons and electromagnetic effects from the Summit Framework", Submitted to Nuclear Fusion, November 2002.

LD15. Y. Ishii, M. Azumi, Y. Kishimoto, and J. N. Leboeuf, "Long time scale plasma dynamics driven by the double tearing mode in reversed shear plasmas", Submitted to Nuclear Fusion, November 2002.

LD16. V. I. Sotnikov, B. S. Bauer, J. N. Leboeuf, P. Hellinger, P. Travnicek and V. Fiala, "Development of global magnetohydrodynamic instabilities in z-pinch plasmas in presence of nonideal effects", Submitted to Phys. Plasmas, November 2002.

Conference Proceedings 
LD17. R. D. Sydora, J. N. Leboeuf, J. M. Dawson, V. K. Decyk, M. W. Kissick, C. L. Rettig, T. L. Rhodes, G. R. Tynan, J. Boedo, J. Ongena, A. Messiaen, and P. E. Vandenplas, "Nonlinear gyrokinetic simulations of ion turbulence in impurity seeded and high density toroidal plasmas," in Proceedings of the 18th IAEA Fusion Energy Conference, Sorento, Italy, 4-10 October 2000, FEC2000, Paper IAEA-CN-77/THP1/27(IAEA, Vienna, 2000).

http://www.iaea.or.at/programmes/ripc/physics/fec2000/pdf/thp1_27.pdf

LD18. V. K. Decyk and D. E. Dauger, "Supercomputing for the masses: a parallel Macintosh cluster", Proceedings of 4th International Conference on Parallel Processing and Applied Mathematics, Naleczow, Poland, 9-12 Sept. 2001, Lecture Notes in Computer Science, Vol.2328, p. 10-22, Springer Verlag, Berlin, 2002.

LD19. Chengkun Huang, Decyk V, Shuoqin Wang, Dodd ES, Chuang Ren, Mori WB, Katsouleas T, Antonsen T Jr., " QuickPIC: a parallelized quasi-static PIC code for modeling plasma wakefield acceleration", PACS2001, Piscataway, NJ, USA, Proceedings of the 2001 Particle Accelerator Conference, vol.5, pp.4005-4007, IEEE, 2002.

LD 20. Vladimir I. Sotnikov, Volodymyr Makhin, Bruno S. Bauer, Petr Hellinger, Pavel Travnicek, Vladimir Fiala, and Jean-Noel Leboeuf, "Hybrid Simulations of Current-Carrying Instabilities in ZPinch Plasmas with Sheared Axial Flow", Beams/DZP2002, Albuquerque, New Mexico, USA, 23 28 June 2002, in DENSE Z-PINCHES: 5th International Conference on Dense Z-Pinches, Jack Davis, Christopher Deeney, Nino R. Pereira, Editors, AIP Conference Proceedings 651, p. 396-399, American Institute of Physics, College Park, MD, USA, December 2002.

http://proceedings.aip.org/proceedings/confproceed/651.jsp

LD21. G. J. Morales, F. S.Tsung, and J.-N. Leboeuf, "Dynamics of a Supersonic Plume Moving Along a Magnetized Plasma", ICPP 2002: International Congress on Plasma Physics, 15 Jul 2002 19 Jul 2002, Sydney, NSW, Australia.

http://www.ise.canberra.edu.au/ICPP2002/

LD22. V. I. Sotnikov, B. S. Bauer, J. N. Leboeuf, P. Hellinger, P. Travnicek, and V. Fiala, "Development of Z-pinch Instabilities in Plasma with Axial Magnetic Field", in Proceedings of ICENES 2002-11th International Conference on Emerging Nuclear Energy Systems, Sheraton Old Town Hotel, Albuquerque, New Mexico U.S.A, September 29-October 4, 2002, Compiled and Edited by Barbara L. Daniels and Deborah G. Cole, p. 255-259, University of New Mexico, December 2002.

http://www.unm.edu/ isd/icenes/ICENES_PrelimProgram.pdf

LD23. T. L. Rhodes, J.-N. Leboeuf, D. Ross, J. Candy, G. R. McKee, R. Bravenec, E. J. Doyle, R. J. Groebner, W. A. Peebles, R. D. Sydora, L. Zeng, and G. Wang, "Quantitative Comparisons of DIII-D Turbulence Measurements to Gyro-Kinetic and Gyro-Fluid Turbulence Simulations", Paper EX/C4-1Rb, 19th IAEA Fusion Energy Conference, FEC 2002, 14 - 19 October 2002, Lyon, France.

LD24. Y. Ishii, M. Azumi, Y. Kishimoto, and J. N. Leboeuf, "Long Time Scale Plasma Dynamics Driven by the Double Tearing Mode in Reversed Shear Plasmas", Paper TH/5-2, 19th IAEA Fusion Energy Conference, FEC 2002, 14 - 19 October 2002, Lyon, France. 
LD25. S. E. Parker, Y. Chen, B. I. Cohen, A. M. Dimits, W. M. Nevins, D. Shumaker, V. K. Decyk, and J. N. Leboeuf, "Large-Scale Gyrokinetic Turbulence Simulations with Kinetic Electrons from the Summit Framework", Paper TH/P1-13, 19th IAEA Fusion Energy Conference, FEC 2002, 14 - 19 October 2002, Lyon, France

Invited Communications

LD26. R. D. Sydora, "Nonlinear dynamis of isolated and overlapping microscopic and mesoscopic magnetic islands in high temperature plasmas", Paper NI2.4, 42nd Annual Meeting of the APS Division of Plasma Physics combined with the 10th International Congress on Plasma Physics October 23 - 27, 2000, Québec City, Canada, Bulletin of the American Physical Society (BAPS) 45, 257 (2000b).

LD27. V. K. Decyk and C. D. Norton, "Modernizing FORTRAN77 Legacy Codes", Conference on Computational Physics 2000, Gold Coast, Australia, December 2000.

LD28. J. N. Leboeuf, "Small University Groups in the US Fusion Theory and Computing Program: The UCLA Experience", Fusion Energy Sciences Advisory Committee (FESAC) Theory Panel Meeting, University of California at Los Angeles (UCLA), Wednesday January 31, 2001.

LD29. J. N. Leboeuf, T. L. Rhodes, J. M. Dawson, V. K. Decyk, and R. D. Sydora, "Comparisons of Nonlinear Global Gyrokinetic Calculations with Experimental Results for DIII-D-Like Discharges", Oral Presentation 1D02, Monday April 2, 2001, 2001 International Sherwood Fusion Theory Conference, Santa Fe, New Mexico, USA, April 2-4, 2001.

LD30. J. N. Leboeuf, T. L. Rhodes, J. M. Dawson, V. K. Decyk, and R. D. Sydora, "Comparisons of Nonlinear Global Gyrokinetic ITG Calculations with Experimental Results for DIII-D-Like Discharges", Contributed Talk, Core III, Thursday May 17, 2001, 2001 US-European Transport Task Force Meeting, Fairbanks, Alaska, USA, May 16-19, 2001.

LD31. V. K. Decyk, "Code modernization and object oriented programming with FORTRAN90", Summit Gyrokinetic Framework Kick-Off Meeting, Doubletree-Del Mar, San Diego, July 24-25, 2001.

LD32. V. K. Decyk, "Supercomputing for the Masses: A Parallel Macintosh Cluster", Keynote Address, International Conference on Parallel Procesing and Applied Mathematics, Naleczow, Poland, September 2001.

LD33. T. L. Rhodes, J.-N. Leboeuf, R. D. Sydora, R. J. Groebner, E. J. Doyle, G. R. McKee, W. A. Peebles, C. L. Rettig, L. Zeng, and G. Wang, "Comparison of turbulence measurements from DIII-D L-mode and high performance plasmas to turbulence simulations and models", Invited Talk UI1.005, 43rd Annual Meeting of the APS Division of Plasma Physics, October 29 - November 2, 2001, Long Beach, CA.

LD34. J. N. Leboeuf, V. Decyk, A. Dimits, and D. Shumaker "Realistic Geometry Extension of Summit", Summit Team Meeting, Sunday April 21, 2002, Rochester, N. Y.

LD35. V. K. Decyk, " Project AppleSeed: Supercomputing for the Rest of Us", US-Japan Workshop on Simulations of Plasmas", UCLA Campus, Los Angeles, CA, May 16-17, 2002. 
LD36. J. N. Leboeuf, V. Decyk, A. Dimits, and D. Shumaker, "Progress with pg3eq_nc, the general geometry version of the gyrokinetic particle-in-cell code pg3eq", Summit Gyrokinetic Workshop, Hyatt Islandia, San Diego, CA, September 19-20, 2002. 